\title{
Advancing Cancer Therapy
}

\author{
Cancer therapies have evolved considerably in recent decades, substantially improving the quality of life and \\ survival of patients with cancer. In this issue, we launch our Series on Cancer Therapy, exploring current paradigms \\ and recent advances and challenges in this field, through specially commissioned articles.
}

T he earliest evidence of cancer treatment can be traced back to an ancient Egyptian medical text, written around $3000 \mathrm{BC}$ and known widely as the 'Edwin Smith Papyrus', that described the cauterization of breast tumors for which, according to the text, there was no cure. The situation is very different now, as, depending on breast cancer subtype, stage and demographic factors, the 5-year survival rates for this disease can surpass $90 \%$ in developed countries. For cancer types that are responsive to therapy, including certain subtypes of breast, blood and prostate malignancies, patients now face the management of a chronic disease, rather than a fatal one, owing to the rapid advances in clinical oncology over recent decades. Similarly, the prognosis for several other cancer types has also been improving. For example, patients with melanoma, which used to be considered a deadly disease, have much better prospects thanks to the breakthroughs in targeted and immune-based therapies.

These advances reflect the focus placed on cancer research and oncology by governments, funders and research institutes across the globe over the past several decades. In the USA, 2021 marks the 50-year anniversary of the signing of the National Cancer Act into law, which marked the beginning of a concerted effort to address cancer as a leading cause of death in the USA at the federal level. The National Cancer Program that arose from this initiative resulted in a profound institutional reorganization within the National Institutes of Health, with the overarching goal of developing the infrastructures required 'for the treatment, cure, and elimination of cancer'. Other countries and international agencies also adopted cancer-focused initiatives over the years, including, for example, the PRIME scheme of the European Medicines Agency, which supports the development of medicines that target an unmet medical need, including cancer, through accelerated planning, evaluation and approval processes.

Thus, substantial progress has been made across first-line cancer therapy modalities. Surgery continues to be a first-line treatment for many cancer types, but it now includes precision and minimally invasive surgery, molecular imaging support and, more recently, robot- or artificial intelligence-assisted surgical procedures. The clinical use of one of the most widely used treatment modalities, chemotherapy, has been improved through better dosing regimens, neoadjuvant or adjuvant administration, and combination therapies. Similarly, radiation oncology has been advanced through precision radiotherapy. First-line recommendations depend on the cancer type and stage at diagnosis, and have continued to be modified as new therapeutic modalities have become available. The advent of targeted therapy and immunotherapy has revolutionized the treatment of cancer, especially with the development and availability of sophisticated diagnostic and molecular characterization technologies. Among these, '-omics' techniques stand out for increasingly enabling a more precise and granular molecular characterization of cancer types and subtypes and the identification of biological correlates of response to specific therapies, thereby enriching the roster of biomarkers at the disposal of clinicians.

Targeted therapies have swiftly taken a prominent position in cancer research and clinical oncology in recent decades, thanks to the molecular insights into oncogenic processes and mechanisms gained from fundamental research and technological development. A key example of how basic research on oncogenic alterations translated into substantial clinical benefits for a large number of patients is BCR-ABL1 tyrosine-kinase inhibitors for chronic myeloid leukemia. The first BCR-ABL1 tyrosine-kinase inhibitor was discovered through drug screens in 1992, and in 2001 it became the first-line therapy with long-term remission rates for BCR-ABLdriven chronic myeloid leukemia'; second-generation tyrosine-kinase inhibitors, rationally designed to circumvent acquired resistance, earned approval from the US Food and Drug Administration as frontline therapies only a decade later. More recently, the announcement of the two first-in-class inhibitors of the mutant kinase KRAS G12C was a milestone in the decades-long efforts to study and treat tumors bearing these, up-to-now considered undruggable, KRAS mutations ${ }^{2}$. However, not every effort in precision oncology and targeted therapy is yielding similarly positive results, especially given the issue of adaptive and acquired resistance, a complication of therapy that a large part of the cancer-research community is striving to address. It should also be noted that advances in sophisticated cancer therapeutics are sometimes associated with a high financial burden for patients, a pressing societal issue tied to the complexities of addressing the challenge of cancer ${ }^{3}$.

In light of the progress made so far and the goals and challenges ahead, we are pleased to launch in this issue of Nature Cancer a Series on Cancer Therapy comprising specially commissioned Review, Perspective, News and Comment articles and a collection of relevant primary research articles published in Nature Cancer. The series is housed in a dedicated page on the Nature Cancer website and will be continually updated with additional content from key opinion leaders discussing novel therapeutic opportunities, the path to drug discovery, and how these advances are transforming clinical practice.

Our series launches with two Review articles that focus on different but important aspects of cancer treatment. Whereas substantial achievements have been witnessed in the treatment of primary tumors, progress has been more modest for metastatic disease. Yibin Kang and colleagues discuss the clinical challenge of treating metastatic disease, and how preclinical and mechanistic knowledge accumulated over the years is being translated into tangible clinical benefits for disseminated disease $e^{4}$. The authors also discuss the challenges of running clinical trials for metastatic disease, and the different degrees of success of clinical trials in the metastatic setting. In a separate Review, Frank McCormick and colleagues discuss the multiple and complex links between oncogenic KRAS-one of the most frequently mutated and, as noted above, hard-to-target cancer drivers-and metabolism, highlighting the potentially targetable vulnerabilities that arise at the 
interface of the two ${ }^{5}$. Although various aspects of targeting KRAS-dependent cancer metabolism have been explored extensively in preclinical settings, ongoing and future clinical trials will hopefully shed light on the translatability of these approaches to the clinic.

Despite the many milestones achieved in cancer treatment, much remains to be addressed. In future issues we will present additional pieces focusing on a breadth of topics under this theme, including key pathways deregulated in cancer, such as EGFR or PI3K, and ongoing clinical approaches for preventing and bypassing therapy resistance. Future issues will also discuss progress in radiotherapy, immunotherapy and therapy combinations, as well as new therapeutic modalities, such as bispecific antibodies, and innovative drug-development approaches through the implementation of artificial intelligence.

Through this selection of commissioned and primary research publications, we aim to underscore how much cancer therapy has advanced over the past several decades, which goals need to be prioritized, and the challenges that should be overcome to continue improving quality of life and outcomes for patients with cancer. We thank our authors and referees for their valuable contributions and hope that our readers will find this Series on Cancer Therapy informative and inspiring.

Published online: 24 March 2021

https://doi.org/10.1038/s43018-021-00192-x

References

1. Druker, B. J. et al. N. Engl. J. Med. 344, 1038-1042 (2001).

2. Bar-Sagi, D., Knelson, E. H. \& Sequist, L. V. Nat. Cancer 1, 25-27 (2020).

3. Gruber, K. Nat. Cancer 1, 1136-1139 (2020)

4. Esposito, M. et al. Nat. Cancer https://doi.org/10.1038/s43018021-00181-0 (2021).

5. Mukhopadhyay, S. et al. Nat. Cancer https://doi.org/10.1038/ s43018-021-00184-x (2021). 